\section{Gräsertablette wirkt nachhaltig}

\author{
Verschiedene Studienergebnisse haben bereits auf eine mögliche \\ Langzeitwirkung der sublingualen Immuntherapie hingewiesen. Nun \\ liegen die ersten Daten zur Wirksamkeit eine Gräsertablette im \\ ersten Jahr nach abgeschlossener Hyposensibilisierung vor.
}

\begin{abstract}
eit dem Jahr 2005 untersuchen Forscher aus 43 europäischen Zentren im Rahmen einer randomisierten, doppelblinden und plazebokontrollierten Phase-III-Studie Wirksamkeit und Sicherheit der Immuntherapie mit der Gräsertablette Grazax ${ }^{\circledR}$ (Allergengehalt 75.000 SQ Phleum pratense pro Einzeldosis). Einbezogen in die Studie waren erwachsene Patienten mit einer mäßigen bis schweren, durch Gräserpollen bedingten Rhinokonjunktivitis, die randomisiert über drei Jahre hinweg einmal täglich Grazax ${ }^{\circledR}$ oder Plazebo sublingual applizierten. Nach der Therapiephase konnten 257 Patienten, das sind etwa 65\% der Teilnehmer der aktiven Studienphase, über ein weiteres Jahr nachbe-
\end{abstract}

obachtet werden. Diese Daten wurden jetzt ausgewertet.

Bereits im ersten aktiven Behandlungsjahr war der Effekt der SQ-standardisierten Gräsertablette statistisch signifikant gewesen und hatte sich bis ins dritte Behandlungsjahr weiter verbessert. Ein Jahr nach Beendigung der Behandlung blieb die deutliche Reduktion der Rhinokonjunktivitissymptome in der Verumgruppe im Vergleich zu der Plazebogruppe bestehen (Differenz 26\%, $\mathrm{p}<0,001)$, ebenso die des Verbrauchs an Akutmedikamenten (Differenz 29\%, $\mathrm{p}=0,022)$. Klare Unterschiede ergaben sich außerdem bei den symptom- und medikationsfreien Tagen - den sogenannten „well days“ - und der Lebens- qualität. Auch der unter der sublingualen Immuntherapie gestiegene Serumtiter von $\mathrm{IgG}_{4}$ und die IgE-blockierende Aktivität waren ein Jahr nach Absetzen der Gräsertablette noch nachweisbar.

Fazit: Die Dreijahresbehandlung mit der Gräsertablette Grazax ${ }^{\circledR}$ führt zu einer deutlichen Verbesserung der klinischen Symptomatik und der Lebensqualität von Heuschnupfen-Patienten, begleitet von erkennbaren immunologischen Veränderungen. Das Niveau der erzielten Ergebnisse wurde auch ein Jahr nach Absetzen der Behandlung noch gehalten. Dies weist auf einen krankheitsmodifizierenden Effekt der spezifischen Immuntherapie mit der Gräsertablette hin.

\section{Durham SR et al. Long-term clinical} efficacy in grass pollen-induced rhinoconjunctivitis after treatment with SOstandardized grass allergy immunotherapy tablet. J Allergy Clin Immunol 2010; 125: 131-38

\section{Spezifische Immuntherapie rechnet sich}

\section{Eine allergische Rhinitis im Kindesalter beeinträchtigt nicht nur die Gesundheit und Lebensqualität, sie führt auch zur verstärkten Inanspruchnahme medizinischer Leistungen. Ob die Investition in eine spezifische Immuntherapie (SIT) sich medizinisch wie finanziell lohnen könnte, untersuchte eine retrospektive Studie.}

A us der Datenbank einer Gesundheitsfürsorge-Institution in Florida ermittelten Forscher alle unter 18-Jährigen, die nach der Erstdiagnose einer allergischen Rhinitis eine SIT mit mindestens zwei Injektionen erhalten hatten. Jedem Patienten wurden bis zu fünf
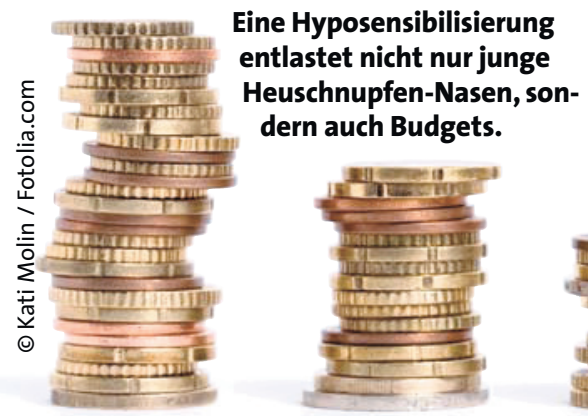

Kontrollpersonen gegenübergestellt, die bezüglich der Diagnose allergische Rhinitis sowie in Alter und Geschlecht vergleichbar waren, aber keine Hyposensibilisierung erhalten hatten.

Die 2.771 mit einer SIT behandelten Patienten verursachten im Lauf von 18 Monaten nach Diagnosestellung signifikant weniger Kosten für medizinische Leistungen insgesamt als die 11.010 Kontrollen, die keine SIT erhalten hatten (3.247 vs. 4.872 US-\$Patient, $\mathrm{p}<0,001)$. Der Unterschied war großenteils auf Einsparungen im ambulanten Bereich zurückzuführen (1.107 vs. $2.626 \$, p<0,001)$. Auch die übrigen Medikamenten- kosten waren durch die SIT erniedrigt (1.108 vs. $1.316 \$, \mathrm{p}<0,001)$. Die SIT selbst hatte im Median 628 \$ gekostet. Der finanzielle Vorteil machte sich erstmals drei Monate nach Beginn der SIT bemerkbar und vergrößerte sich im Lauf des weiteren Beobachtungszeitraums stetig. In der Häufigkeit und Dauer stationärer Aufenthalte und den entsprechenden Kosten für die Krankenhausbehandlung unterschieden sich die beiden Patientengruppen nicht.

Fazit: Eine SIT bei Kindern und Jugendlichen mit allergischer Rhinitis rechnet sich nach dieser Studie rasch und anhaltend. Die Autoren rufen daher zu einer stärkeren Nutzung dieser Therapie auf. Das ist für die USA von besonderer Bedeutung, weil dort die SIT im Vergleich zu Europa eine untergeordnete Rolle spielt.

$f k$

Hankin CS et al. Allergen immunotherapy and health care cost benefits for children with allergic rhinitis: a large-scale, retrospective, matched cohort study. Ann Allergy Asthma Immunol 2010; 104: 79-85 